\title{
Factors affecting repositioning policy compliance: an integrative review
}

Abdulkareem Iblasia, ${ }^{a, b}$ Yupin Aungsurocha,*, Joko Gunwan ${ }^{a}$

aDepartment of Nursing, King Saud Medical City, Riyadh 11143, Saudi Arabia

${ }^{b}$ Faculty of Nursing, Chulalongkorn University, Pathumwan, Bangkok 10330, Thailand

Received: 31 April 2020; Accepted: 26 June 2020; Published: 20 March 2021

Abstract: Objective: To explore the factors affecting nurses' compliance with repositioning policy. Methods: An integrative review was conducted following the Whittemore and Knafl methodology to identify the problem related to repositioning policy compliance. We searched the following databases: Coherence Wounds Group Specialized Register (Jan 1997 to Jun 2019), Ovid MEDLINE (Jan 1997 to Jun 2019), EBSCO CINAHL (Jan 1997 to Jun 2019), and Clinical Key database (Jan 2014 to Oct 2018).

Results: The review revealed three factors that influence repositioning compliance: nurse-related factors, patient-related factors, and Environment-related factors.

Conclusions: These factors directly impact one another and, in turn, influence the compliance of nurses to the repositioning policy. However, there is no evidence currently available that explains the collective impact of these factors and how they interact to affect repositioning policy compliance. Nevertheless, all these factors are important and should be considered to enhance and further improve the quality of nursing care and adherence to the repositioning policy.

Keywords: integrative review $\bullet$ factors influence $\bullet$ nursing behavior $\bullet$ policy $\bullet$ repositioning compliance

(c) Shanxi Medical Periodical Press.

\section{Introduction}

The pressure ulcer advisory panels defined a pressure ulcer/injury as "localized damage to the skin and/or underlying tissue usually over a bony prominence, as a result of pressure, or pressure in combination with shear, but may also occur from medical devices or other objects." 1,2

A lack of repositioning compliance results in vessels being constricted under gravitational pressure, which stops or critically minimizes the amount of blood passing through to the target tissues. ${ }^{3}$ This soon results in irreversible tissue damage,${ }^{4}$ which might occur even earlier in overweight patients. ${ }^{5}$ Irreversible tissue damage then initiates the formation of a pressure injury. ${ }^{6,7}$ Pressure ulcers reduce patients' quality of life ${ }^{8}$ and place a burden on hospital resources as the prevention cost is much less than the treatment cost. ${ }^{9}$ According to the current understanding of pressure formation, relieving pressure from the bony prominences or sites of medical devices is the cornerstone in preventing pressure ulcer formations. $3,4,10,11$

Experts consider patient repositioning to be a significant pressure ulcer prevention measure ${ }^{1,2,12}$ that is

How to cite this article: Iblasi A, Aungsuroch Y, Gunwan J. Factors affecting repositioning policy compliance: an integrative review. Front Nurs. 2021;1:7-22. 
defined as an effort to regularly modify patient posture..$^{13}$ In hospital settings, repositioning is a nursing responsibility ${ }^{14}$ and hospitals have created repositioning policies to help nurses achieve proper repositioning. ${ }^{15}$ Nurses are accountable for complying with such policies to prevent the incidence of pressure ulcers. Repositioning compliance refers to the performance of repositioning in manner of such quality and frequency to achieve the pressure relief.

However, it has been observed that compliance with repositioning policy in nursing units is low. ${ }^{16,17}$ Low compliance refers to situations in which nurses are unable to achieve the required quality or frequency in repositioning patients according to policy instructions. For instance, in Saudi Arabia, the national guideline states that hospitals must clarify what pressure ulcer preventions are in place, including their repositioning policy ${ }^{18,19}$ but clinical evidence indicates that nurses typically do not comply with the repositioning policy. ${ }^{15,20}$ Internationally, the situation is similar. In the United States, reports indicate that only $40 \%$ of patients in need of repositioning were treated appropriately. ${ }^{21}$ This was also the case as observed in India where approximately only $30 \%$ of patients received the required repositioning. ${ }^{22}$ Results of studies were similar in Belgium, ${ }^{23}$ Sweden, ${ }^{24}$ Egypt, ${ }^{25}$ China, ${ }^{26}$ Australia, ${ }^{27}$ Hong Kong, China, ${ }^{28}$ Saudi Arabia, ${ }^{15,20}$ and the Netherlands. ${ }^{29}$ Therefore, low repositioning compliance is an observed phenomenon among nurses in hospitals across cultures. Therefore, this article aimed to identify factors affecting repositioning policy compliance among nurses at the clinical level to support stakeholders in understanding repositioning compliance phenomena and to aid in the design of suitable changes to evidence-based repositioning policy. To achieve this goal, we followed the methodology used by Whittemore and $\mathrm{Knafl}^{30}$ to answer the review question: "What are the factors affecting repositioning compliance among nurses in clinical units?" The review methodology consists of five steps: problem identification, review of the studies, evaluation of the data, data analysis, and presentation of findings.

\section{Methods}

\subsection{Problem identification}

Although nurses' compliance in performing repositioning should be $100 \%$ compliance with repositioning policy ranges between $13.9 \%{ }^{23}$ and $75 \% .{ }^{14,31}$ The current low compliance rate increases the possibility that at-risk patients will develop pressure ulcers ${ }^{32}$ as well as highlights the low quality of nursing care that patients are subjected to..$^{8,9}$ Therefore, leaders and stakeholders are obligated to identify the reasons for this low compliance.

\subsection{Literature search}

\subsubsection{Types of studies}

This review included manuscripts published between 1997 (when the European Pressure Ulcer Advisory Panel [EPUAP] began) and 2019 and included all studies that investigated factors influencing nurses' repositioning compliance. Cohort studies were included if they outlined or reported factors associated with repositioning compliance among nurses. Qualitative studies and quality projects were included if the studies presented repositioning compliance among nurses as a concern. The search was not limited to any specific methods of addressing repositioning compliance as a primary or secondary outcome of processes indicated for preventing pressure ulcers. Studies that investigated repositioning compliance among non-nursing staff or in a non-pressure ulcer prevention context were not included.

The following electronic databases were searched: Coherence Wounds Group (Jan 1997 to Jun 2019), Ovid MEDLINE (Jan 1997 to Jun 2019) based on the Saudi Commission for Health Specialties (SCFHS) access, EBSCO CINAHL (Jan 1997 to Jun 2019), Clinical Key database (Jan 2014 to Oct 2018), and the reference sections of retrieved studies. During the search, the authors applied the same terms to all databases, which are as follows: repositioning compliance, positioning compliance, repositioning, change in patient positioning, change patient position, pressure ulcer prevention, pressure ulcer prevention policy/guideline, pressure injury prevention, pressure ulcer injury policy/guideline, bedsores prevention, and decubitus ulcer prevention.

\subsubsection{Types of participants}

Studies reporting repositioning compliance in any healthcare facility that requires nurses to comply with repositioning intervention to prevent pressure injury formation were included in this article. No limitations were established based on the type of hospital, scope of services, or nursing home.

\subsubsection{Types of interventions and outcome measures}

No limits were placed on the types of interventions applied to improve repositioning compliance or pressure ulcer management. The study criteria included studies that presented repositioning compliance as an outcome or process indicator for pressure ulcer management. 


\subsubsection{Description of studies}

The initial search identified 923 citations (776 from electronic databases and 147 from the reference check). A total of 122 duplicated reports were subsequently excluded. Of the remaining 801 studies, 497 reports were excluded because they did not assess pressure injury prevention. Thereafter, 304 studies were reviewed by general reading, and an additional 215 studies were excluded for failing to meet the inclusion criteria (191 evaluated repositioning compliance among non-nursing personnel, and 24 did not analyze repositioning compliance as a nursing intervention). The entire text of the remaining 89 studies was reviewed. A total of 30 studies did not discuss factors related to repositioning policy compliance as a concern, and the authors differed in their opinions about five studies. After consultation, these studies were excluded from the analysis. A total of 54 studies met the criteria, as presented in the PRISMA chart (Figure 1).

\subsection{Data evaluation}

\subsubsection{Selection of studies}

The authors reviewed the selected studies separately. The evaluation revised the titles of studies retrieved from the database search. Complete reports of all potential studies that matched the above-mentioned criteria were prepared on an Excel sheet for ease of access and then arranged in tables. In cases of disagreement, a senior author was asked to adjudicate on the inclusion of studies. The authors listed the causes for rejection and were not blinded to the study authorship. The authors evaluated the references used in the

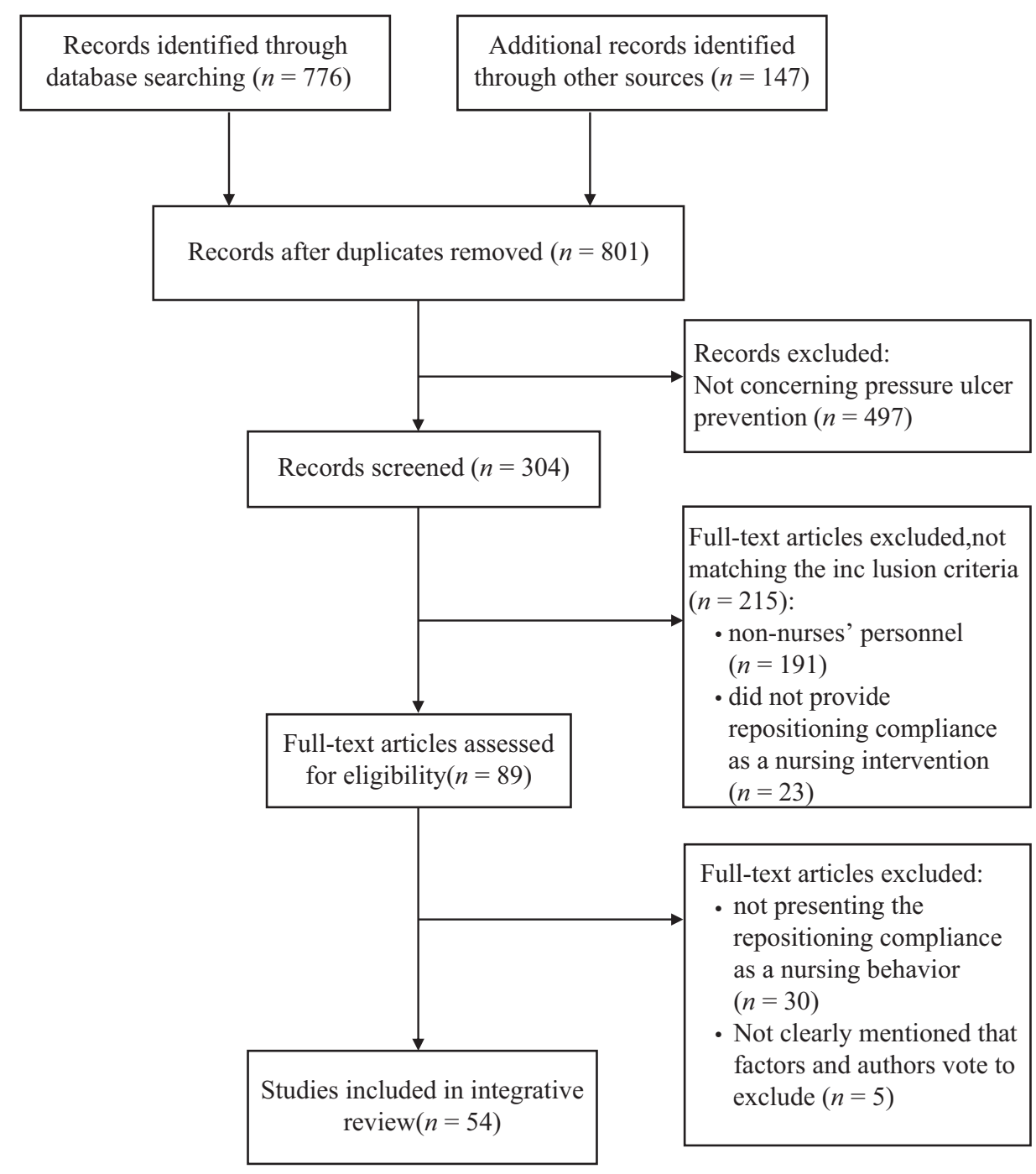

Figure 1. PRISMA chart. 
revised studies to verify any further reports that met the selection criteria.

\subsubsection{Risk of bias assessment}

Two authors autonomously evaluated the risk of bias in the selected studies. For clinical trials, the authors implemented the Cochrane Collaboration's tool. ${ }^{33}$ The authors ranked the studies as low risk, high risk, and unclear (unknown) risk of bias. ${ }^{33}$ They also applied critical appraisal for qualitative studies to evaluate the quality level. Each qualitative study was evaluated for level of credibility, transferability, dependability, and confirmability. ${ }^{34}$ The studies were ranked on a three-point scale (high, moderate, and low) for each of the aforementioned items. ${ }^{34}$

A total of 54 studies matched the inclusion criteria. These included 4 studies of randomized controlled trials, 5 qualitative studies, 14 quasi-experimental studies, 3 retrospective studies, and 15 cross-sectional studies, as well as 4 prospective design studies, 2 observational studies, 4 quality projects, 2 triangulation studies, and 1 longitudinal study. The studies show a low to moderate level of bias, as presented in Table 1; however, no study was excluded from the analysis due to bias risks.

\subsubsection{Data extraction and management}

After evaluating all retrieved studies and determining which ones were relevant based on the inclusion/ exclusion criteria, the authors individually extracted data using a predesigned data collection sheet designed for this study based on the recommendations of Souza, Silva, and Carvalho ${ }^{35}$ The designed table summarizes the following information from the included studies, as described in the criteria in Polit and Beck ${ }^{36}$ type of publication, methodological characteristics, and level of evidence. The data extraction and analysis were based on the content of each study. The datasheet contained the information is listed in Table 1.

\subsection{Data analysis}

All studies agreed that repositioning policy compliance is a mandatory nursing practice but presented various factors and different effects with respect to repositioning compliance. Twelve factors were found to influence repositioning policy compliance. These factors were repeated 97 times across all the studies reviewed, as presented in Table 2. The most frequently mentioned variables included nursing attitudes and quality projects (15 studies), while other factors include nursing knowledge, skills, staffing, teamwork, direct managerial feedback, nursing empowerment, nursing assignments, availability of repositioning assistance devices, and patients' characteristics. The summary descriptions for all studies are presented in Table 1.

\subsubsection{Nursing-related factors}

The studies present nursing characteristics that influence nurses' compliance with repositioning policy and are referred to as nursing-related factors. These factors include nursing knowledge, nursing skills, and nursing attitudes toward repositioning. Nurses require a minimum level of nursing knowledge to comply with repositioning policy. ${ }^{37-39}$ Studies present nursing knowledge as the level of information that nurses have regarding the need for repositioning policy compliance in pressure ulcer prevention.

The nursing repositioning knowledge includes general identification of the characteristics of repositioning as an intervention. ${ }^{40,41}$ It also includes an understanding of the assessment tools to determine at-risk patients. ${ }^{23}$ However, the studies do not standardize repositioning knowledge definitions but agree that the knowledge resource should be consistent with the international pressure ulcer guidelines. ${ }^{1,2}$

The second factor is nursing skill. Nursing skill in performing repositioning is divided into two parts: the ability of nurses to perform the procedure $e^{42,43}$ and the absence of any physical restraint that would prevent them from complying with repositioning policy. ${ }^{44}$ A nurse's ability to perform the procedure indicates that the nurse has the required competencies to "reposition" the patients based on the predefined steps in the policy. ${ }^{45}$

The third individual factor is the nurses' attitude. The attitude of nurses was defined as a critical factor among studies that investigated nurses' compliance with repositioning policy. ${ }^{23,46}$ Researchers assume that attitude is the main factor contributing to nurses' compliance with repositioning policy. Nurses have profound antagonistic feelings regarding repositioning policy compliance because repositioning - in their view - is a procedure that should be delegated to students and practical nurses, and it does not need to be performed by a registered nurse. ${ }^{47,48}$ These negative attitudes also weaken the focus of nurses to follow up on compliance with repositioning after delegation.

\subsubsection{Patient-related factors}

Patients' characteristics also affect compliance with repositioning policy. ${ }^{41}$ For instance, patients with excess weight, ${ }^{41}$ old age,$^{24}$ and pressure ulcers ${ }^{5}$ receive less repositioning than patients who are bedridden with the same conditions. Patients on medications that treat 


\begin{tabular}{|c|c|c|c|}
\hline Author and country & Population and sample & Design (level of evidence) & Findings \\
\hline $\begin{array}{l}\text { Alexander et al. } \\
\text { USA }\end{array}$ & $\begin{array}{l}\text { Nurses in nursing homes } \\
N=2\end{array}$ & Observational (VI) & $\begin{array}{l}\text { Face-to-face communication is increased when an IT } \\
\text { system with a low level of sophistication that includes the } \\
\text { repositioning decision is used to prevent pressure injuries } \\
\text { and a higher level of care is provided when a highly } \\
\text { sophisticated IT system is used. }\end{array}$ \\
\hline
\end{tabular}

Ali et al. Egypt Nurses in a hospital $N=83 \quad$ Longitudinal study (VI)

Amon et al. USA

Medical-surgical telemetry patients

Sample: One unit with 32 beds

Angmorterh et al.

UK

Athlin et al. ${ }^{5}$

Sweden

Beeckman et al. Belgium

Beeckman et al Belgium

Behrendt et al. UK

Hospitalized critical care patients $N=422$

Experimental group $=213$

Control group $=209$

Black and Maegley Hospitalized patients

USA

23 beds in a medical-surgical unit

Cameron et al. UK Nurses in the NHS

Chaboyer et al. Hospitalized patients

Australia
$N=1,600$
Pre-post Intervention (III)

Prospective experimental (III)

Qualitative content analysis (VI)

Cross-sectional study (VI)

RCT (II)

Prospective control study (IV)

Quality improvement project (III)

Quality improvement project (III)

Clustered randomized control trial (II)
The changes in nurses' performance regarding pressure injury prevention, which includes repositioning, resulted from changes in the administrative focus and the involvement of nurses in the responsibilities.

The incidence of pressure ulcer PU was reduced as a result of compliance with pressure injury prevention policies, including repositioning.

The pressure placed on volunteers when moving the patient on the radiology trolley is high, and the settings are not convenient for the repositioning of the patient during the diagnostic procedures in radiology.

Pressure injury prevention compliance, including repositioning, is associated with:

i. nurses' perceptions of the patient's physical and psychological conditions and nurses' perceptions of the level of cooperation from patients;

ii. Responsibilities and commitments among nurses toward repositioning and pressure injury prevention;

iii. Nursing knowledge and skills;

iv. Cooperation and teamwork among the nurses in the unit;

v. Availability of policies and routine care in the hospital; and

vi. Availability of repositioning equipment and required pressure redistribution supplies.

i. Only $13.9 \%$ of high-risk patients received appropriate prevention, including repositioning compliance.

ii. The level of knowledge among nurses is lower than the expected while attitudes are high.

iii. Pressure injury prevention, including repositioning, correlates with attitudes, but no independent correlation is observed between knowledge and prevention.

After implementing the supported clinical decision, nurses knowledge and positive attitudes increased but did not result in significant changes in their compliance toward pressure injury prevention, including repositioning, while increased compliance with the pressure injury prevention policy was observed for patients in wheelchairs.

Following the implementation of specific equipment that provides continuous and consistent visual feedback for nurses, nurses exhibit better compliance with repositioning. No further education or any further policy changes were implemented.

The project demonstrates changes in the nurses' compliance with pressure injury prevention, including repositioning, after the application of a new policy, forms, and follow-up system.

The project presents the experiment performed in the NHS to provide the pressure ulcer prevention program online. The project assumes an increase in pressure ulcer prevention compliance, including repositioning.

The study shows a decrease in the incidence of pressure ulcers and an increase in prevention following the application of the pressure injury prevention care program, including repositioning, but statistically significant differences were not observed between the overall compliance of nurses in both groups. 


\begin{tabular}{lll}
\hline Author and country & Population and sample & Design (level of evidence) \\
\hline \hline Choi et al. USA & Nurses in critical care facilities & Quality improvement \\
$N=15$ & project (III) \\
& \\
& \\
& \\
\end{tabular}

Courvoisier et al. Switzerland

Co x USA

Cub ukcu Turkey

Cyriacks and Spencer USA

Dellefield and

Magnabosco USA

Feng et al. China

Nurses in hospital $N=275$

Fossum et al.

Norway

Gunningberg et al Sweden

Patients in nursing homes $N=2,671$

Hospitalized critical care patients $N=347$ units $N=786$

Hospitalized patients

Nurses in hospitals $N=16$$$
N=15
$$
medical ward $N=190$
Patients in nursing home care 32 beds in a pulmonary unit

project (III)

Triangulation study design (III)

Pre-/post-intervention quasi-experimental study (III)

Quasi-experimental study (III)

Patients in the geriatric/internal design (IV)

Pragmatic randomized trial (III)
Finding

The low compliance of nurses is associated with:

i. Nurses' attitudes toward a lack of responsibility for repositioning compliance;

ii. A reduced sense of personal agency;

iii. Barriers including a lack of time, lack of staff, and attitudes that patients have a very high acuity for preventing pressure ulcers, including the priority of repositioning; and

iv. Leadership and interdisciplinary cooperation are supportive elements.

Cross-sectional study $(\mathrm{VI}) \quad$ The study reported relations between the prevalence of pressure ulcers and the type and size of nursing homes.

Retrospective correlational The study concludes that the predictability of the Braden scale is questionable. The most reliable predictions were based on patient mobility and friction/shear. Many risk factors have been identified empirically; however, a consensus on the most important risk factors is lacking. $18.7 \%$ of patients developed a pressure ulcer. A pressure ulcer is preventable.

A pressure ulcer is associated with prolonged hospitalization.

Cross-sectional study (VI) The study concludes that pressure ulcer development is associated with the Braden score, weak nutritional conditions, and chronic illnesses.

The identification of these factors during the initial assessment of patients supports proper pressure ulcer prevention measures.

The study shows increased repositioning compliance following the redistribution of the nursing staff and creation of a turning team from nurses on duty.

i. Evidence that links individual nursing factors with pressure ulcer prevention compliance, including repositioning, is limited

ii. Nurses, in general, had a positive attitude toward repositioning.

iii. Nurses' perceptions of recognition are low.

iv. Higher performance feedback is associated with greater compliance.

v. Nurses prefer patients who can give positive feedback

vi. Nurses in the same organization describe their knowledge, attitudes, and beliefs about pressure ulcer prevention, including repositioning, differently.

vii. Communication and workload perceptions are associated with compliance and require further investigation

Improvements in the knowledge and attitudes of nurses toward pressure ulcer prevention and repositioning through an awareness campaign are associated with higher levels of compliance and a lower incidence of pressure ulcers.

i. Nurses' compliance with all care related to pressure ulcers, including repositioning, improved after applying a software system.

ii. The application of the software provides the education and support required for nurses to decide on the required care. i. No significant changes in pressure ulcer incidence were observed after applying the pressure mapping system.

ii. Applying the pressure mapping system increased the nurses' repositioning compliance as they received feedback.

iii. The study presents the need for further investigations in this area. 


\begin{tabular}{|c|c|c|c|}
\hline Author and country & Population and sample & Design (level of evidence) & Findings \\
\hline Hanna et al. USA & $\begin{array}{l}\text { Nurses working in different } \\
\text { organizations } N=429\end{array}$ & Cross-sectional study (VI) & $\begin{array}{l}\text { i. The study explores a group of silent factors that affect } \\
\text { nurses' decisions to reposition patients. } \\
\text { ii. The silent factors related to patients are patient weight, } \\
\text { which is negatively associated with repositioning } \\
\text { compliance. } \\
\text { iii. Patients' cooperation has positive relations with } \\
\text { repositioning compliance. } \\
\text { iv. Teamwork between nurses. }\end{array}$ \\
\hline $\begin{array}{l}\text { Hartmann et al. } \\
\text { USA }\end{array}$ & $\begin{array}{l}\text { Nurses in a nursing home } \\
N=23\end{array}$ & Qualitative study (VI) & $\begin{array}{l}\text { Staff provided the following indirect impressions of the } \\
\text { facilitators and barriers associated with pressure ulcer } \\
\text { prevention: } \\
\text { i. Structure: A formal structure such as a team or } \\
\text { committee will advance the practice. } \\
\text { ii. The establishment of organizational priorities will } \\
\text { enhance the performance. } \\
\text { iii. An improvement in culture. } \\
\text { iv. Clear roles and responsibilities. } \\
\text { v. Communication strategies. } \\
\text { vi. Staff and clinical practices. }\end{array}$ \\
\hline
\end{tabular}

Hall and Clark

USA

Kalisch et al. USA surgical ICU $N=100$

Nurses in hospitals $N=4,086$

Patients and relatives in hospitals $N=729$

Källman and

Suserud Sweden

Källman et al.

Sweden
Pre- and Post-intervention (III)

Cross-sectional study (VI)

Cross-sectional study (VI)

Cross-sectional study (VI)

Non-experimental, observational study (VI)
The study concludes that the device reduces the repositioning burden among nurses, increases repositioning compliance, and reduces the HAPU among the selected units.

i. A lack of proper nursing care was common among all hospitals.

ii. Job title, shift work, absenteeism, perceived staffing adequacy, and patient workload were significantly associated with a lack of proper nursing care, including repositioning.

iii. Repositioning is one of the top five tasks that most directly affect patients' outcomes by preventing pressure ulcer development.

Patients with pressure ulcers (they did not receive the proper prevention measures, including repositioning) reported higher rates of missed nursing care in the following areas:

1. Overall lack of proper nursing care.

2. Lack of nursing communication and

3. Time to response by nurses (all of which were significantly associated with pressure ulcers).

i. Nurses have good knowledge.

ii. Nurses also generally have a positive attitude toward care.

iii. Nurses have a negative perception of the organizational support system for providing care.

iv. Nurses mentioned the following barriers for proper pressure ulcer prevention, including repositioning: lack of time, lack of staff, a shortage or lack of equipment and facilities in the organizations, and a lack of related policies.

v. Nurses perceived many uncooperative ill patients.

The study concludes that repositioning compliance is associated with:

i. The existence of a PU during the day. If patients presented with a pressure ulcer, nurses did not intend to maintain compliance with repositioning during the day shift, while the situation was not the same during the night shift.

ii. Patients with cancer received higher repositioning compliance during the night shift.

iii. Nurses in hospitals were more compliant with repositioning than nurses in nursing homes.

iv. The use of sheets and other equipment reduced nurses' compliance with repositioning.

v. The patient's general activity, moisture level, cognitive dysfunction, and use of psycholeptic medications all negatively correlated with repositioning compliance. Thus, nurses did not intend to be compliant with this category of patients. 


\begin{tabular}{lll}
\hline Author and country & Population and sample & Design (level of evidence) \\
\hline \hline $\begin{array}{l}\text { Knibbe et al. } \\
\text { Netherlands }\end{array}$ & $\begin{array}{l}\text { Patients in nursing homes } \\
N=13\end{array}$ & $\begin{array}{l}\text { Quasi-experimental pre- } \\
\text { post-intervention study (III) }\end{array}$ \\
& & \\
& & \\
Kwong et al. Hong & $\begin{array}{l}\text { Patient and nurses in nursing } \\
\text { Kong }\end{array}$ & $\begin{array}{l}\text { Action research of three } \\
\text { main steps (III) }\end{array}$
\end{tabular}

Lavallée et al. UK

Nurses in the hospital $N=25$

Qualitative study (VI)

Lu et al. China

Bedridden patients in a gynecological unit $N=150$

Mallah et al. Lebanon

Meesterberends et al.

Mendoza et al. Saudi Arabia

Moore and Price Ireland
Patients in nursing homes $N=547$ Germany and The Netherlands

Inpatient units $N=17$

Nurses in a hospital $N=121$
RCT - Two armed (II)

Quasi-experimental prepost-intervention study (III)

Prospective multicenter cohort study (IV)

Pre- and post-intervention (III)

Cross-sectional study (VI)
Findings

The case reports did not reveal a significant difference in nurses' compliance with repositioning before and after implementing repositioning facilities. However, the study presents the importance of the availability of this device in reducing nurses' working-related hazards.

The changes in nurses' behavior in complying with repositioning resulted from the factors listed below:

i. Nursing empowerment and training. "They increase the feelings of responsibility belonging to the nurse through participation, which affects repositioning compliance."

ii. It is an organizational development model to lower pressure ulcer incidence.

iii. The application of protocols enhanced practice.

The study defined seven domains that interact as barriers or facilitators in manipulating nurses' behavioral compliance, which are listed below.

i. The barriers are 1 - knowledge, 2 - physical skill, 3 social influences, and 4 - environment and resources.

ii. The facilitators are 1 - interpersonal skills, 2 environmental context, 3 - beliefs about capabilities, 4 - beliefs about consequences, and 5 - social and professional roles.

The report shows a significant increase in the implementation of pressure ulcer prevention measures (including repositioning) after applying the protocol. Significant reductions in pressure ulcer incidence were also observed after applying the clinical guideline.

i. Changes in HAPU were observed after applying the bundle.

ii. Repositioning compliance positively correlates with changes in nursing assignments to create a champion.

iii. Repositioning compliance is associated with patient age (younger, better compliance; low length of stay, better compliance)

iv. The overall repositioning compliance rate is $75.62 \%$ and the compliance rate for high-risk patients is $65.2 \%$.

i. More PUs occurred in The Netherlands than in Germany

ii. The factors that explained the differences between Germany and The Netherlands are nurses' perceptions of 1 - dementia, 2 - analgesic use, 3 - use of transfer aids, 4 - repositioning compliance, 5 - availability of a tissue viability nurse, and 6 - quality control.

iii. All factors were better in The Netherlands (except internal quality control), while the pressure ulcer rate and compliance were lower. Therefore, the main factor associated with better compliance in Germany is internal quality control.

An awareness campaign increases nurses' compliance in preventing pressure ulcers.

i. Nurses have positive attitudes toward repositioning.

ii. Nurses intend to practice pressure ulcer prevention, including repositioning, without a scientific rationale.

iii. Nurses perceived a lack of time and lack of staff as barriers to pressure ulcer prevention, including repositioning

iv. Registered nurses had positive attitudes toward the importance of repositioning and other prevention measures.

v. A positive attitude does not influence or change nurses' practice regarding repositioning compliance.

vi. No relation is observed between positive attitudes and practice.

vii. When nurses perceive the situation as a shortage, repositioning is not a priority.

viii. Nurses perceive other nursing responsibilities as much more important than pressure ulcer prevention, including repositioning.

ix. No clear role of knowledge in influencing nursing compliance is identified, although educational resources are limited for nurses. 


\begin{tabular}{|c|c|c|c|}
\hline Author and country & Population and sample & Design (level of evidence) & Findings \\
\hline $\begin{array}{l}\text { Moya-Suárez et al. } \\
\text { Spain }\end{array}$ & $\begin{array}{l}\text { Nurses working in hospitals } \\
N=249\end{array}$ & Cross-sectional study (VI) & $\begin{array}{l}\text { i. The questionnaire is valid for predicting nurses' } \\
\text { compliance with the recommendations for pressure } \\
\text { ulcer prevention, including repositioning. } \\
\text { ii. Nurses' attitudes play a major role in their decision to } \\
\text { implement measures that prevent pressure ulcers. }\end{array}$ \\
\hline Peterson et al. USA & Patients in a hospital $N=23$ & Observational study (VI) & $\begin{array}{l}\text { i. The method nurses used to perform the repositioning } \\
\text { is called the triple jeopardy area under pressure, which } \\
\text { leads to a pressure ulcer. } \\
\text { ii. The study questioned the skill of nurses performing the } \\
\text { repositioning. } \\
\text { iii. In addition, the study presents the need for a further } \\
\text { analysis of the concept of repositioning itself. As the } \\
\text { procedure of repositioning requires further reforms } \\
\text { for supine left-right, the performance of all these } \\
\text { procedures currently does not employ the proper } \\
\text { repositioning techniques. }\end{array}$ \\
\hline
\end{tabular}

$\begin{array}{lll}\begin{array}{l}\text { Renganathan et al. } \\ \text { India }\end{array} & \begin{array}{l}\text { Hospitalized critical care } \\ \text { patients } N=40\end{array} & \begin{array}{l}\text { Prospective, non- } \\ \text { randomized, multiphase, } \\ \text { multicenter trial (III) }\end{array}\end{array}$

Rich et al. USA Hospitalized patients $N=269$

Saliba et al. UK Patients in nursing homes $N=834$

Samuriwo UK Nurses in nursing homes $N=16$ Samuriwo UK $\quad \begin{aligned} & \text { Nurses in nursing homes } \\ & N=16\end{aligned}$

Still et al. USA

Strand and Lindgren Sweden
Hospitalized critical care patients in a surgical ICU $N=507$

Nurses in intensive care units $N=146$
Observational study (VI)

Retrospective analysis (III)

Qualitative grounded theory (VI)

Qualitative grounded theory "Reanalyzed data" (IV)

Pre- and post-intervention quasi-experimental study (III)

Cross-sectional study (VI)

Schutt et al. USA
Hospitalized patients $N=138$
Quasi-experimental prepost-intervention study (III)
The use of a continuous repositioning monitoring system increases nurses' compliance with the repositioning protocols.

i. Repositioning compliance is low, with $53 \%$ of patients in need receiving the required repositioning.

ii. Differences in the incidence of pressure ulcers are not observed between patients who received repositioning in less than or greater than two hours.

iii. Patients with pressure ulcers on admission had better repositioning policy compliance than those at-risk but without pressure ulcers

i. A significant difference exists between facilities, even if they are applying the same policies and protocols.

ii. Pressure injury prevention guideline compliance is a problem in $\mathrm{NH}$.

iii. Nursing homes differ in their level of compliance; further investigations are needed.

i. A link exists between the value of nurses and pressure ulcer prevention compliance, including repositioning.

ii. Repositioning is usually delegated to students and health care assistants.

iii. Pressure ulcer prevention is perceived as less critical than other nursing interventions, such as doctors' orders.

iv. With less follow-up performed by nurses, nurses intend to delegate the repositioning task.

The reanalysis of the data also concludes a substantial role for multidisciplinary teamwork and nursing empowerment in compliance with pressure ulcer prevention measures, including repositioning.

Significant changes in repositioning compliance were observed after the staff were redistributed to establish a turning team in the unit.

i. Nurses' attitudes toward pressure injury prevention, including repositioning compliance, are good.

ii. A significant difference in knowledge is observed between registered nurses and practical nurses.

iii. Nurses attribute a low level of compliance to a lack of time $(57.8 \%)$, the severity of the patient's condition (28.9\%), or lack of the required equipment (35.5\%)

iv. The study stresses the need for further improvements in the knowledge of pressure injury prevention, including repositioning.

i. Nurses significantly respond to the availability of a continuous feedback system.

ii. Nurses have a low level of compliance with repositioning, but the application of public follow-up significantly increases compliance. 


\begin{tabular}{ll}
\hline Author and country & Population and sample \\
\hline \hline Sving et al. Sweden & nurses in hospitals $N=9$
\end{tabular}

Sving et al. Sweden nurses in hospitals $N=9$

Sving et al. Sweden

Hospitalized patients $N=825$

Hospitalized patients and residents in nursing homes $N=21,378(H)$ $N=15,579(\mathrm{NHs})$

Tayyib and Coyer Saudi Arabia

Tayyib et al. Saudi Arabia

Ünver et al. Turkey

Nurses in a hospital $N=101$

Hospitalized patients with pressure ulcers $N=133$ Surgical unit: 58

Medical unit: 73

Cancer unit: 2

Design (level of evidence) Findings

Triangulation study design (III)

Descriptive crosssectional study (VI)

Cross-sectional study (VI)

Clustered randomized control trial (II)
Findings

The article aimed to describe how RNs perform, document, and reflect on pressure ulcer prevention compliance, including repositioning.

ii. Repositioning is one of the items observed and monitored by the authors and is marked as a caring culture or social issue.

iii. RNs show limited attention to pressure ulcer prevention, including repositioning.

iv. Nurses generally perform repositioning for other reasons than pressure ulcer prevention.

v. A lack of knowledge among nurses is noted.

vi. A communication deficit exists between RNs and ANs, leading to missed scheduled repositioning.

vii. Nurses over-trusted nurses assistants in pressure injury prevention.

viii. RNs have a proper attitude toward pressure ulcer prevention, including repositioning, but they do not intend to perform the procedure alone.

i. A low total number of nursing staff is associated with low repositioning compliance.

ii. Patients with a higher score on risk tools received higher compliance from nurses than other patients.

iii. Nurses perceived that older patients would require higher repositioning compliance.

iv. Repositioning compliance in geriatric units is higher than in other units.

v. Nurses should not exclusively rely on the records when evaluating repositioning compliance.

The study defines the differences in pressure ulcer prevention, including the repositioning compliance between two countries as related to:

i. Educational programs

ii. Policies and protocols

iii. Special follow-up team

iv. Follow-up system

i. The study applied OMRU (the Ottawa Model) to facilitate the successful dissemination of a new pressure injury prevention care bundle, which includes repositioning.

ii. The study was conducted in two hospitals in Saudi Arabia and shows that the use of care bundles and protocols reduces the incidence of PU and increases pressure injury prevention compliance, including repositioning, as the nurses already participated in creating the protocol.

iii. Modification of the durations for repositioning from 2 to 3 hours increases compliance.

i. Repositioning compliance is only influenced by the unit norms, but not any patient's condition.

ii. The unit policy and administrative follow-up are the only factors contributing to behavioral modification among nurses in the critical care unit. No relations with the Braden score or any further redaction tools are associated with nurses' compliance with pressure ulcer prevention, including repositioning.

Cross-sectional study (VI) i. Nurses' attitudes toward pressure ulcer prevention, including repositioning, is the main factor related to compliance.

ii. Nurses' attitudes toward pressure ulcer prevention, including repositioning, are positive.

Retrospective cohort study (IV)

The study reviewed the long-term effects of applying protocols on the compliance level. The study supports the hypothesis that nurses' long-term compliance will increase when a bundle of care or protocols to organize the care is available. 


\begin{tabular}{|c|c|c|c|}
\hline Author and country & Population and sample & Design (level of evidence) & Findings \\
\hline \multirow[t]{2}{*}{ Weiner et al. Israel } & Nurses in a hospital $N=48$ & Post-test (III) & $\begin{array}{l}\text { The study classifies the participants into three groups in } \\
\text { which repositioning was performed with different levels } \\
\text { of assistance for patients with different weights. The } \\
\text { equipment plays an important role in reducing low back } \\
\text { pain among nurses, which improves compliance. }\end{array}$ \\
\hline & & & $\begin{array}{l}\text { The study concluded that the sliding sheet is the only } \\
\text { assistive equipment that allows all nurses to perform all } \\
\text { required repositioning. }\end{array}$ \\
\hline Wogamon USA & $\begin{array}{l}\text { Nursing assistants in hospitals } \\
N=33\end{array}$ & Non-randomized trial (III) & $\begin{array}{l}\text { i. On-the-job training for nursing assistants increases } \\
\text { compliance. Therefore, a lack of compliance is related } \\
\text { to knowledge and skills. } \\
\text { ii. Increased documentation and compliance are } \\
\text { observed after the educational sessions. }\end{array}$ \\
\hline
\end{tabular}

Table 1. Summary of available studies that present the motivations for repositioning compliance.

\begin{tabular}{lc}
\hline Factor & Frequency of studies \\
\hline \hline Individual factor - Attitude & 15 \\
Individual factor - Knowledge & 10 \\
Individual factor - Skill & 5 \\
Environmental -Managerial follow-up & 3 \\
Environmental- Staffing & 15 \\
Environmental - Teamwork & 7 \\
Environmental factor - Equipment & 12 \\
Environmental factor - Quality projects & 15 \\
Environmental - Digital visualization & 3 \\
Patient factor - Age & 2 \\
Patient factor - Weight & 2 \\
Patient factor - Medical condition & 8 \\
Total & 97 \\
\hline
\end{tabular}

Table 2. Frequency of factors influencing repositioning compliance reported among the studies.

psychosis also receive less repositioning. ${ }^{5}$ While these two clinical conditions should not influence nurses in terms of less compliance with repositioning policy, patients with oncological disorders receive higher repositioning policy compliance. ${ }^{24}$

The studies did not determine whether this behavior was related to nurses' perceptions regarding the importance of repositioning policy compliance or a negative attitude of care. ${ }^{24}$ These findings pave the way for further follow-up and in-depth investigations. Therefore, nurses' managers should follow up on vulnerable patient groups regarding the nature of nursing care and ensure repositioning compliance.

\subsubsection{Environment-related factors}

Environmental factors are variables that reflect on the nurses' surroundings. These factors comprise of the digital visualization feedback, teamwork, staffing, direct managerial feedback, quality improvement projects, and repositioning assistant aids. These are tangible and non-tangible supporting factors for improving repositioning policy compliance.

The first factor is digital visualization feedback. This is an electronic system that follows up on repositioning progress by the assigned nurses and presents the repositioning performance on a public screen in the unit. $21,22,49$ Thus, repositioning policy compliance for each staff member is in public view and the digital visualization feedback significantly improves compliance. ${ }^{21,22,49}$ However, repositioning compliance improved only when nurses were provided with visualizations of their levels of performance in a public place. There were no studies in which nurses' compliance levels improved if compliance was not made publicly visible. Thus, the visualization of nurses' level of performance in a public place significantly improved compliance.

The second factor is nursing teamwork. Repositioning policy compliance requires synchronized human efforts from two to three nurses to complete the patient shifts, safely establish a new posture,$^{50}$ and frequently repeat the procedure based on the policy. Teamwork is a compulsory factor in establishing repositioning policy compliance..$^{51}$ However, studies present the absence of teamwork as a barrier to ensuring repositioning policy compliance. ${ }^{52}$ Furthermore, the shared responsibilities inherent in teamwork play a role in reminding ${ }^{53}$ and motivating nurses ${ }^{21}$ to comply with repositioning policy. Therefore, the relationship between the clinical team and repositioning policy compliance is vital and logical.

Third, an appropriate number of nursing staff to provide proper workload distribution had a positive effect on repositioning policy compliance. For example, in the study by Cyriacks and Spencer, ${ }^{40}$ modifying the nursing staffing plan improved the repositioning compliance. The staffing plan was modified by assigning nurses to repositioning teams; these teams were responsible for the repositioning of all at-risk patients. Furthermore, as per the investigation in Still et al., ${ }^{54}$ the manager 
redistributed nurses to organize a team that focused on performing the repositioning with no further assignments. However, these interventions required employing more nurses in the units or withdrawing nurses from other units and assigning them to repositioning tasks only, but this would only create a heavier workload on the remaining nurses. ${ }^{49}$ Therefore, modifying the staffing plan to include the required number of nurses would allow these nurses to adhere to the repositioning policy as well as complete other nursing tasks.

Direct managerial feedback on repositioning policy compliance also enhances nurses' performance ${ }^{56}$ and thus, represents the fourth factor. Evidence indicates that when frontline managers make more effort to follow up on repositioning policy compliance, nurses demonstrate a higher compliance level. ${ }^{25,57}$

The fifth factor is quality improvement projects. A quality improvement project is a multidisciplinary effort aimed at enhancing or improving the practice related to pressure ulcer prevention in the hospital. Studies demonstrate that an increase in repositioning policy compliance occurs in institutions that have quality improvement projects. ${ }^{48,51,56}$ Hospitals with quality improvement plans to reduce pressure ulcer formations follow and monitor nursing behavior in repositioning compliance. These effects are observed with all types of quality projects either at the higher ${ }^{26,58}$ or at the lower administrative level. ${ }^{29,40}$

Sixth, repositioning aids are devices or tools that support nurses during the repositioning procedure. ${ }^{59}$ The absence of these aids was mentioned as a barrier for ensuring a good repositioning policy in two qualitative studies and led to complaints from the nurses. ${ }^{47,49}$ However, other evidence contradicted any role of the presence of a repositioning aid in enhancing nurses' compliance..$^{35,56}$ In addition, while such aids are likely to play an essential role in facilitating compliance, it might be a secondary variable in these situations.

Overall, this article presents several factors that influence nurses to comply with the repositioning policy. These factors do not affect the nurses' behaviors separately, but rather simultaneously. In essence, the factors are not independent of each other but go hand in hand. However, there is no available evidence to consider the effects of these factors on each other. For instance, enhancing nursing knowledge will have an impact on nursing attitudes; how one factor affects another would be reflected on the compliance of the nurses to the repositioning policy. In another example, organizations that work to enhance the nursing knowledge among nurses may be focused on other things at the same time such as quality improvement projects or the purchase of repositioning aids. Therefore, the compliance of the nurses would be representative of how these factors affect/influence each other. However, there are no available studies that present these relations at the clinical level or how these actions interact together to shape the repositioning policy compliance phenomena, but the available knowledge about its effects may suffice.

A nursing manager will not be able to organize a straightforward evidence-based practice that does not take all of these factors into consideration. This article presents groups of factors in different categories. Each of these factors affects each other in various ways, but there is no method available that can identify or calculate the effect of these factors collectively. Hence, there is a need for further investigation to understand the synergy between these factors and how they interact to influence nurses' compliance with the repositioning policy.

\subsection{Implications}

This article presents several issues related to repositioning compliance. First, it is a significant challenge in many hospitals. Also, repositioning compliance is a multifactorial phenomenon, ${ }^{60}$ and several factors influence the nursing compliance level. Hospitals need to interact with the current challenges in the immediate future. The hospital intervention should assure the presence of systematic efforts to address poor compliance. Specifically, only single interventions or focusing on specific aspects will not necessarily lead to the desired changes. Thus, changing the current situation requires an understanding of all of these factors and organizing the change interventions that take all of these issues into consideration. Nursing management must consider all these factors in organizing a straightforward evidence-based practice to enhance repositioning compliance.

\subsection{Presentation}

The authors designed a chart that presents all these factors that relate to repositioning policy compliance (Figure 2).

\section{Conclusions}

Several factors influence repositioning policy compliance. This integrative literature review of 54 studies reveals three main types of factors that influence nurses' compliance with repositioning policy: nurse-related factors, patient-related factors, and environment-related factors. The nurse-related factors include nurses' knowledge, skills, and attitudes toward repositioning compliance. Patient-related factors include patients' age, weight, the presence of a pressure ulcer, and certain medical diagnoses, such as cancer or the use of medications to 


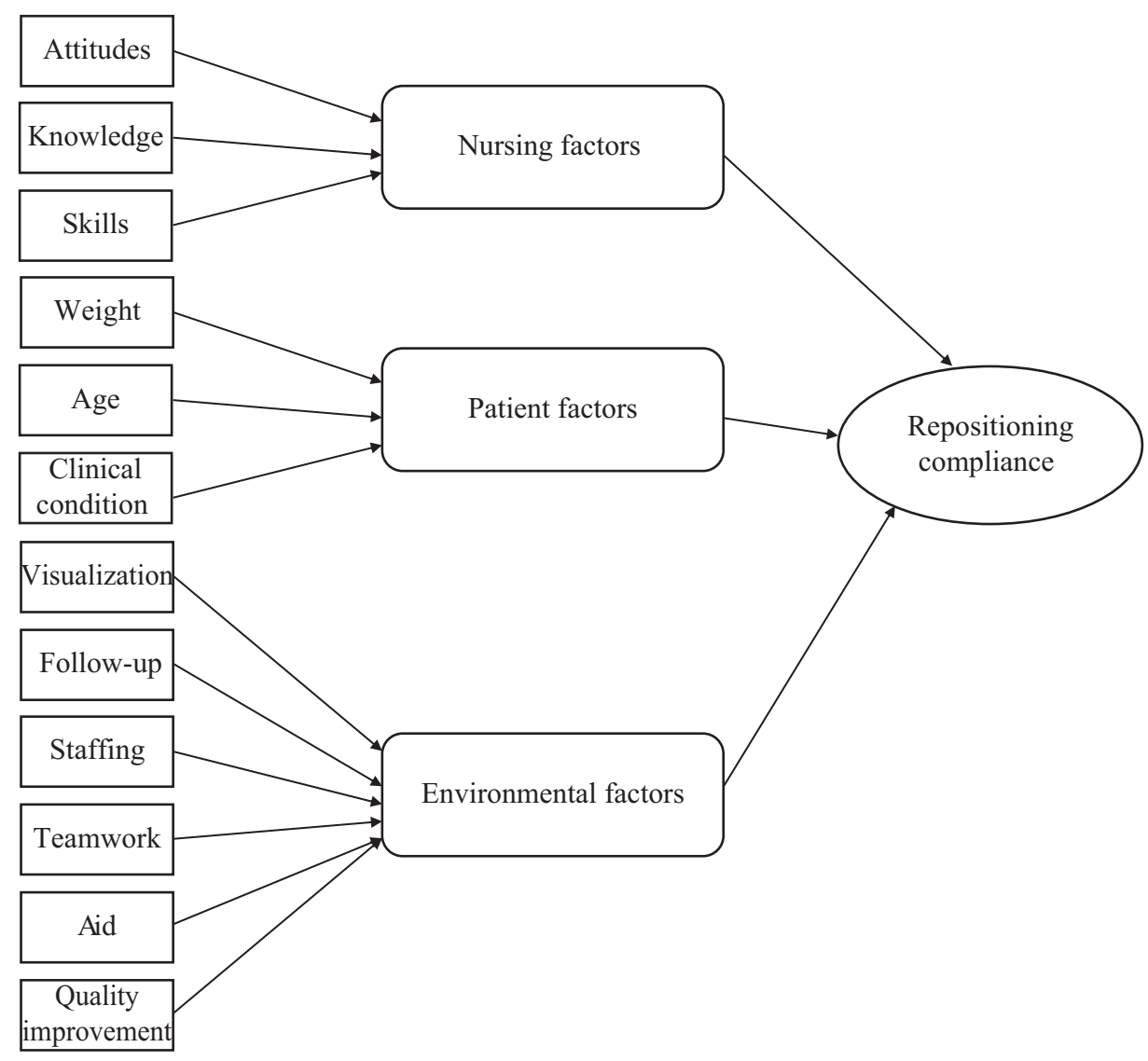

Figure 2. Presentation of the factors.

treat psychosis. The environment-related factors include digital visualization feedback, teamwork, staffing, direct managerial feedback, availability of quality improvement projects, and availability of repositioning supporting aids. The conclusions of this article demonstrate the necessity of including all these factors to overcome the challenges of developing evidence-based programs to improve the repositioning compliance of nurses.

\subsection{Limitations}

This integrative literature review did not exclude any study on repositioning policy compliance that was

\section{References}

1. European Pressure Ulcer Advisory Panel, National Pressure Injury Advisory Panel and Pan Pacific Pressure Injury Alliance (EPUAP/NPIAP/ PPPIA). Prevention and Treatment of Pressure Ulcers/Injuries: Clinical Practice Guideline: The International Guideline; 2019. published in English; however, other relevant studies that were either not published in English or not available for review may have been overlooked. Thus, repositioning policy compliance might be modified by additional influential factors that are not presented in this review.

\section{Ethical approval}

Ethical issues are not involved in this article.

\section{Conflicts of interest}

All contributing authors declare no conflicts of interest.

2. National Pressure Injury Advisory Panel, European Pressure Ulcer Advisory Panel and Pan Pacific Pressure Injury Alliance (NPUAP/EPUAP/PPPI). Prevention and Treatment of Pressure Ulcers: Clinical Practice Guideline: Cambridge Media; 2014. 
3. Gefen A. Reswick and Rogers pressure-time curve for pressure ulcer risk. Part 1. Nurs Stan. 2009;23:64, 66, 68.

4. Gefen A. Reswick and Rogers pressure-time curve for pressure ulcer risk. Part 2. Nurs Stan. 2009;23:40-44

5. Athlin E, Idvall E, Jernfält M, Johansson I. Factors of importance to the development of pressure ulcers in the care trajectory: perceptions of hospital and community care nurses. J Clini Nurs. 2010;19:2252-2258.

6. Collier MZ. Etiology and risk factors. Scie prac pres ulcer mana. London: Springer; 2006:27-36.

7. Redelings MD, Lee NE, Sorvillo F. Pressure ulcers: more lethal than we thought? Adv Skin Wound Care. 2005; 18:367-372.

8. Rutherford C, Brown JM, Smith I, et al. A patientreported pressure ulcer health-related quality of life instrument for use in prevention trials (PU-QOL-P): psychometric evaluation. Heal Qual Life Outcomes. 2018;16:227.

9. Demarré L, Van Lancker A, Van Hecke A, et al. The cost of prevention and treatment of pressure ulcers: a systematic review. Int J Nurs Sci. 2015;52:1754-1774.

10. Schwartz D, Gefen A. The biomechanical protective effects of a treatment dressing on the soft tissues surrounding a non-offloaded sacral pressure ulcer. Int Wound J. 2019;16:684-695.

11. Gefen A. The future of pressure ulcer prevention is here: detecting and targeting inflammation early. J Eur Wound Manage Assoc. 2018;19:7-13.

12. Berman AT, Shirless S, Geralyn F. Kozier-Erb's Fundamentals of Nursing: Concepts, Process, and Practice. USA: Pearson Education Inc; 2016.

13. Hampton S. Could lateral tilt mattresses be the answer to pressure ulcer prevention and management? $\mathrm{Br} J$ Community Nurs. 2017;22:S6-S12.

14. Rich SE, Margolis D, Shardell M, et al. Frequent manual repositioning and incidence of pressure ulcers among bed-bound elderly hip fracture patients. Wound Repair Regener. 2011;19:10-18.

15. Tayyib N, Coyer F. Translating pressure ulcer prevention into intensive care nursing practice. J Nurs Care Qual. 2017;32:6-14.

16. Kalisch BJ, Xie B, Dabney BW. Patient-reported missed nursing care correlated with adverse events. Am J Med Qual. 2014;29:415-422.

17. Samuriwo R. The impact of nurses' values on the prevention of pressure ulcers. $\mathrm{Br} J$ Nurs. 2010;19:S4-S14.
18. Shaikh ZM. A Text Book on Patient Care Management. Vol 1. Guntur/Vijayawada, India: KY Publications; 2018.

19. $\mathrm{MOH}$. Pressure Ulcer Protocol (Prevention and Treatment) Mini Heal King Saud Arab. Vol. 1. Ministry of Health - Kingdome of Saudi Arabia; 2019.

20. Tayyib N, Lewis PA, Coyer FM. A prospective observational study of patient positioning in a Saudi intensive care unit. Middle East J Nurs. 2013;7:26-34.

21. Schutt SC, Tarver C, Pezzani M. Pilot study: assessing the effect of continual position monitoring technology on compliance with patient turning protocols. Nurs Open. 2018;5:21-28.

22. Renganathan B, Nagaiyan S, Preejith S, Gopal S, Mitra S, Sivaprakasam M. Effectiveness of a continuous patient position monitoring system in improving hospital turn protocol compliance in an ICU: a multiphase multisite study in India. J Intensive Care Soc. 2018:309-315.

23. Beeckman D, Defloor T, Schoonhoven L, Vanderwee K. Knowledge and attitudes of nurses on pressure ulcer prevention: a cross-sectional multicenter study in Belgian hospitals. World Evidence Base Nurs. 2011;8:166-176.

24. Källman U, Bergstrand S, Ek AC, Engström M, Lindgren $M$. Nursing staff induced repositionings and immobile patients' spontaneous movements in nursing care. Int Wound J. 2016;13:1168-1175.

25. Ali KAG, Helal WESH, Salem FA, Attia H. Applying interdisciplinary team approach for pressure ulcer assessment, prevention and management. Int J Nove Rese Heal Nurs. 2019;5:640-658.

26. Feng H, Li G, Xu C, Ju C. Educational campaign to increase knowledge of pressure ulcers. $\mathrm{Br} J$ Nurs. 2016;25:S30-S35.

27. Chaboyer W, Bucknall T, Webster J, et al. The effect of a patient centred care bundle intervention on pressure ulcer incidence (INTACT): a cluster randomised trial. Int $J$ Nurs Stud. 2016;64: 63-71.

28. Kwong EW, Hung MS, Woo K. Improvement of pressure ulcer prevention care in private for-profit residential care homes: an action research study. BMC Geriatr. 2016;16:192.

29. Meesterberends E, Halfens RJG, Spreeuwenberg $M D$, et al. Do patients in Dutch nursing homes have more pressure ulcers than patients in German nursing homes? A prospective multicenter cohort study. J Am Med Dir Assoc. 2013;14: 605-610. 
30. Whittemore $\mathrm{R}$, Knafl K. The integrative review: updated methodology. J Adv Nurs. 2005;52:546-553.

31. Mallah Z, Nassar N, Badr LK. The effectiveness of a pressure ulcer intervention program on the prevalence of hospital acquired pressure ulcers: Controlled before and after study. Appl Nurs Res. 2015;28:106-113.

32. Marsden G, Jones K, Neilson J, Avital L, Collier M, Stansby G. A cost-effectiveness analysis of two different repositioning strategies for the prevention of pressure ulcers. J Adv Nurs. 2015;71:2879-2885.

33. Higgins PT, Altman DG, Gøtzsche PC, et al. The Cochrane Collaboration's tool for assessing risk of bias in randomised trials. BMJ. 2011;343:d5928.

34. Russell CK, Gregory DM. Evaluation of qualitative research studies. Evidence Based Nurs. 2003;6:36-40.

35. Souza MT, Silva MD, Carvalho RD. Integrative review: What is it? How to do it? Eins (Sao Paulo). 2010;8:102-106.

36. Polit DF, Beck CT. Nursing Research: Generating and Assessing Evidence for Nursing Practice. 8th ed. Philadelphia: Lippincott Williams \& Wilkins; 2008.

37. Wogamon CL. Exploring the effect of educating certified nursing assistants on pressure ulcer knowledge and incidence in a nursing home setting. Ostomy Wound Manage. 2016;62:42-50.

38. Gunningberg L, Sedin IM, Andersson S, Pingel R. Pressure mapping to prevent pressure ulcers in a hospital setting: a pragmatic randomised controlled trial. Int J Nurs Stud. 2017;72:53-59.

39. Strand T, Lindgren M. Knowledge, attitudes and barriers towards prevention of pressure ulcers in intensive care units: a descriptive cross-sectional study. Int Crit Care Nurs. 2010;26:335-342.

40. Cyriacks B, Spencer C. Reducing HAPI by cultivating team ownership of prevention with budget-neutral turn teams. MEDSURG Nurs. 2019;28:48-52.

41. Hanna DR, Paraszczuk AM, Duffy MM, DiFiore LA. Learning about turning: report of a mailed survey of nurses' work to reposition patients. MEDSURG Nurs. 2016;25:219.

42. Källman U, Suserud BO. Knowledge, attitudes and practice among nursing staff concerning pressure ulcer prevention and treatment - a survey in a Swedish healthcare setting. Scan J Caring Sci. 2009;23:334-341.

43. Krapfl LA, Langin J, Pike CA, Pezzella P. Does incremental positioning (weight shifts) reduce pressure injuries in critical care patients? J Wound Ostomy Cont Nurs. 2017;44:319-323.

44. Gonzalez C. Protecting pregnant health care workers from occupational hazards. Work Heal Safe (Formerly AAOHN J). 2011;59:417-420.

45. Cowan DT, Norman I, Coopamah VP. Competence in nursing practice: a controversial concept - a focused review of literature. Nurse Educ Today. 2005;25:355-362.

46. Moore Z, Price P. Nurses' attitudes, behaviours and perceived barriers towards pressure ulcer prevention. J Clin Nurs. 2004;13:942-951.

47. Sving E, Gunningberg L, Högman M, Mamhidir AG. Registered nurses' attention to and perceptions of pressure ulcer prevention in hospital settings. J Clin Nurs. 2012;21:1293-1303.

48. Choi KR, Ragnoni JA, Bickmann JD, Saarinen HA, Gosselin AK. Health behavior theory for pressure ulcer prevention: root-cause analysis project in critical care nursing. J Nurs Care Qual. 2016;31:68-74.

49. Behrendt R, Ghaznavi AM, Mahan M, Craft S, Siddiqui A. Continuous bedside pressure mapping and rates of hospital-associated pressure ulcers in a medical intensive care unit. Am J Crit Care. 2014;23:127-133.

50. Kozier B. Fundamentals of Nursing: Concepts, Process and Practice. USA: Pearson Education; 2009.

51. Duncan KD. Preventing pressure ulcers: the goal is zero. Jt Comm J Qual Patient Saf. 2007;33:605-610.

52. Hartmann CW, Solomon J, Palmer JA, Lukas CV. Contextual facilitators of and barriers to nursing home pressure ulcer prevention. Adv Skin Wound Care. 2016;29:226-238.

53. Yap TL, Kennerly SM, Horn SD, Bergstrom N, Datta S, Colon-Emeric C. TEAM-UP for quality: a cluster randomized controlled trial protocol focused on preventing pressure ulcers through repositioning frequency and precipitating factors. BMC Geriatr. 2018;18:54.

54. Still MD, Cross LC, Dunlap M, et al. The turn team: a novel strategy for reducing pressure ulcers in the surgical intensive care unit. J Am Coll Surg. 2013;216:373-379.

55. Lavallée JF, Gray TA, Dumville J, Cullum N. Barriers and facilitators to preventing pressure ulcers in nursing home residents: a qualitative analysis informed by the Theoretical Domains Framework. Int J Nurs Stud. 2018;82:79-89.

56. Soban LM, Hempel S, Munjas BA, Miles J, Rubenstein LV. Preventing pressure ulcers in 
hospitals: a systematic review of nurse-focused quality improvement interventions. Jt Comm J Qual Patient Saf. 2011;37:245-AP216.

57. Saliba D, Rubenstein LV, Simon B, et al. Adherence to pressure ulcer prevention guidelines: implications for nursing home quality. J Am Geriatr Soc. 2003;51:56-62.

58. Mendoza M, Jovero J, Agbuya M, Tan T. 100 days, $100 \%$ hospital-acquired pressure ulcer-free campaign at a Saudi Arabian rehabilitation facility. Wound Int Middle East. 2014;5:10-13.

59. Knibbe NE, Zwaenepoel E, Knibbe HJJ, Beeckman D. An automatic repositioning system to prevent pressure ulcers: a case series. Br J Nurs. 2018;27:S16-S22.

60. Avsar P, Patton D, O'Connor T, Moore Z. Do we still need to assess nurses' attitudes towards pressure ulcer prevention? A systematic review. J Wound Care. 2019;28:795-806. 